Revista de Economia Política, vol. 42, n 1, pp. 192-206, janeiro-março/2022

\title{
Corporate Venture Capital e Aceleradores Corporativos: diferenças e similitudes
}

\author{
Corporate venture capital and corporate accelerators: \\ differences and similarities
}

MATEUS CHRISTIANO KÖNIG MARTINS $*$
RAFAELA OLIVEIRA PADILHA **
SOLANGE MARIA DA SILVA $* *$

RESUMO: A pesquisa tem como objetivo apontar as diferenças e similitudes entre Corporate Venture Capital (CorpVC) e Aceleradores Corporativos (AC), mecanismos estes de colaboração entre empresas maduras e Startups. A pesquisa apontou que o fenômeno de Aceleradores Corporativos e de Corporate Venture Capital são práticas de inovação aberta, que podem ser semi-institucionalizadas em empresas que possuam a intenção de buscar inovação em ambientes externos. O fato de CorpVC e AC serem baseados em objetivos estratégicos, e não só financeiros, os diferenciam das outras modalidades de investimentos feitas diretamente em empresas, no entanto, percebeu-se que ambas as estratégias têm diferenças cruciais, que, se vistas anteriormente pela empresa madura, podem ser mais bem aplicadas, garantindo o índice de sucesso maior, baseado no propósito e objetivo da Corporação financiante.

PALAVRAS-CHAVE: Corporate venture Capital; Aceleradores Corporativos; Startup.

ABSTRACT: The research aims to point out the differences and similarities between Corporate Venture Capital (CorpVC) and Corporate Accelerators (AC), as mechanisms of collaboration between mature companies and Startups. The research highlighted that the phenomenon of Corporate Accelerators and Corporate Venture Capital are open innovation practices, which can be semi-institutionalized in companies that intend to seek innovation in external environments. The fact that CorpVC and AC are based on strategic objectives, and not only financial ones, differentiate them from other types of investments made

\footnotetext{
* Mestre em Tecnologia da Informação e Comunicação, Universidade Federal de Santa Catarina (UFSC), Brasil. E-mial: mateuscristianomartn@hotmail.com. Orcid: https://orcid.org/0000-0003-1054-5576.

* Mestre em Tecnologia da Informação e Comunicação, Universidade Federal de Santa Catarina (UFSC), Brasil. E-mial: rafaelapadilha_@hotmail.com. Orcid: https://orcid.org/0000-0002-1925-1366.

*** Doutora em Engenharia de Produção, Universidade Federal de Santa Catarina (UFSC), Brasil. E-mail: solange.silva@ufsc.br. Orcid: https://orcid.org/0000.0001.6123.0203. Submitted: 25/March/2019; Approved: 1/June/2021.
} 
directly in companies, however, it was noticed that both strategies have crucial differences, which as previously seen by the mature company, that can be better applied, ensuring the highest success rate based on the purpose and objective of the financing Corporation.

KEYWORDS: Corporate Venture Capital; Corporate Accelerators; Startup.

JEL Classification: M130.

\section{INTRODUÇÃO}

O ecossistema de apoio a Startups vem se expandindo e, com isso, o surgimento de diversas iniciativas que contribuem para seu desenvolvimento. Tal contexto colabora, também, para o aparecimento de um ambiente mais propício ao surgimento de soluções disruptivas aos negócios tradicionais. Assim, muitas corporações têm percebido que não basta investir apenas em pesquisa e desenvolvimento (P\&D) internamente em suas empresas (Weiblen; Chesbrough, 2015; Norris, 2014). As corporações precisam desenvolver conhecimento e experiência de mercado, economia de escala e redes de contato (Mocker; Bielli; Haley, 2015).

Desde a década de 1960, as corporações vêm investindo em Startups (Heinemann, 2015). Nesta modalidade de investimento, aparece a denominada "Corporate Venture Capital” (CorpVC). A literatura apresenta que a institucionalização de técnicas de gestão, por meio do apoio de corporações, pode possibilitar melhores taxas de sucesso de empreendedores. Neste contexto, as corporações estão desenvolvendo modelos de relacionamento com Startups, sem a necessidade de participação societária, permitindo uma avaliação mais ágil de uma quantidade maior de Startups, reduzindo, assim, o custo para inovar. Neste mesmo quadro, entre 2010 e 2011 surgiram, no mundo, as primeiras aceleradoras corporativas de Startups (Heinemann, 2015). Os Aceleradores Corporativos (AC), assim como o CorpVC, oferecem uma abordagem potente para nutrir inovações de empreendimentos empresariais. Os ACs são programas apoiados por empresas maduras de duração limitada, que firmam a capacitação de Startups durante o processo de novos empreendimentos, via tutoria, educação e recursos específicos da empresa (Kohler, 2016). No entanto, as vastas diferenças entre empresas maduras e Startups tornam a colaboração um desafio, seja via CorpVC ou via AC. A presente pesquisa se propõe a identificar as dimensões da CorpVC e da AC, identificando padrões e dissonâncias, a fim de apontar a melhor estrutura para a aquisição de inovação eficaz pela empresa madura.

\section{REFERENCIAL TEÓRICO}

\section{Inovação}

Antes de dar início à conceituação dos principais objetos de pesquisa deste trabalho (Corporate Venture Capital e Aceleradores Corporativos), deve-se apresentar 
o que a literatura aponta sobre o termo que os influencia, na prática e/ou em sua criação, de forma direta ou indireta, denominado Inovação.

Para Tidd, Bessant e Pavitt (1997), a inovação está essencialmente ligada à aprendizagem e mudança, e é frequentemente inédita, arriscada e dispendiosa. É um processo de transformar oportunidades em novas ideias, colocando-as em prática (Tidd; Bessant; Pavitt, 2008). Gunday et al. (2011) consideram inovação como uma evolução e novas aplicações, que tem como objetivo lançar a novidade para o mercado. Ela pode ser constituída como a transformação do conhecimento em valor comercial. A inovação tem grande importância comercial devido ao seu potencial para aumentar a eficiência e a rentabilidade das empresas. Portanto, a inovação desempenha um papel significativo na criação de diferenças de desempenho e concorrência entre as empresas.

\section{Corporate Venture Capital - Capital de Risco Corporativo}

Corporate Venture Capital (CorpVC) é caracterizado como um fundo privado criado e controlado por uma empresa madura, com o propósito de realizar investimentos em projetos de inovação, mais especificamente quando tal investimento é realizado diretamente em uma Startup externa (Chesbrough, 2002). Esta definição exclui investimentos intermediados por gestores de fundos ou em unidades/projetos internos, exceto quando estes últimos já passaram por processos de "spin-off". Apesar de realizarem investimentos financeiros, tais programas não buscam somente retorno monetário à corporação, mas, também, empreendimentos que promovam negócios complementares, ou que alavanquem as vendas de seus produtos (Weiblen; Chesbrough, 2015).

Para Chesbrough (2002), Dushnitsky e Lenox (2005), e Dushnitsky e Shaver (2009), o Capital de Risco Corporativo é um investimento direto de fundos de empresas estabelecidas em Startups, não negociadas publicamente, para atender aos objetivos da empresa investidora, como, por exemplo, a aquisição de conhecimento. Investimentos feitos por terceiros, mesmo em nome da corporação, geralmente não seriam considerados Capital de Risco Corporativo; eles são tipicamente participações minoritárias e, portanto, não devem ser confundidos com fusões ou aquisições. Além disso, a startup tem que ser externa à corporação e não deve já pertencer legalmente à empresa investidora. Além do fluxo de conhecimento, os objetivos das empresas investidoras podem ser múltiplos. Chesbrough (2002) e Hochberg (2015) distinguiram entre objetivos financeiros e estratégicos. Os primeiros podem incluir objetivos de cobertura, bem como retornos financeiros simples, enquanto os últimos podem variar entre promover a estratégia atual (investir em empresas que querem usar os produtos da corporação, por exemplo) até apoiar empresas com produtos complementares. Finalmente, o Capital de Risco Corporativo pode ser uma maneira de uma empresa obter informações sobre as tendências dos mercados emergentes (Dushnitky; Lenox, 2006). As Startups, por outro lado, não apenas se beneficiam do suporte financeiro fornecido, mas, também, podem aproveitar a força 
da marca investidora para adquirir clientes ou ganhar mais rodadas de investimento (Dushnitky; Lenox, 2006; Dushnitky; Shaver, 2009).

Ao longo do tempo, as estruturas CorpVCs têm evoluído. Diversas empresas inovadoras, como Adobe, Intel e Lucent introduziram programas de CorpVC para promover maior inovação e motivar funcionários a se tornarem mais empreendedores e a correrem mais riscos (Chesbrough, 2000). Além dos retornos financeiros que podem ser obtidos com o Capital de Risco Corporativo, esse tipo de empreendimento também oferece benefícios estratégicos como retornos indiretos dessa atividade financeira. Cria-se valor ao oferecer uma possibilidade sobre novas tecnologias e práticas que poderiam impulsionar os próprios esforços de inovação das empresas. Além disso, a varredura do ambiente, em busca de novas tecnologias que possam ameaçar ou complementar os principais negócios, permitirá que o investidor os obtenha por meio de licenciamento ou aquisição. Os empreendimentos que criam produtos e serviços complementares podem aumentar a demanda futura de produtos corporativos, se suportados. Portanto, é inestimável que os capitalistas de risco corporativos, que querem se beneficiar estrategicamente, facilitem a transferência de conhecimento entre o empreendimento e a organização (Dushnitky; Lenox, 2005, 2006). Chesbrough e Tucci (2002) observam que os investimentos em Capital de Risco Corporativo, muitas vezes, alimentam a inovação formal em vez de substituí-la. Porém, as tentativas de aplicar CorpVC encontram, normalmente, sucesso temporário, pois enfrentam várias dificuldades e terminam com a eventual interrupção da iniciativa (Chesbrough, 2000).

\section{Aceleradores Corporativos}

Embora sejam incertas as razões exatas para o surgimento de Aceleradores Corporativos, várias fontes traçaram paralelos com os padrões cíclicos de fundos de Capital de Risco Corporativo e não corporativo. Após a crise da Internet, em 2000, e a crise financeira, em 2008, os capitalistas de risco não apenas reduziram seus investimentos, mas, também, avançaram para Startups em estágio avançado, deixando uma lacuna de financiamento para Startups. Essa lacuna foi parcialmente preenchida pelos programas emergentes de aceleradores não corporativos (Hoffman; Radojevich-Kelley, 2012). Além disso, o fato de aceleradores terem agregado e filtrado Startups em estágio inicial (Hochberg, 2015) foi discutido especialmente durante um período de baixa despesa de capital de risco. No entanto, em vez de desaparecer com a recuperação dos investimentos de capital de risco, após cada crise, os aceleradores sobreviveram e estabeleceram-se como uma nova estrutura no ecossistema de financiamento (Ernst; Young, 2013). Isso foi ainda mais ajudado pelos avanços das tecnologias da informação, que reduziram o custo para lançar novos negócios e tornaram os investimentos comparativamente baixos e aceleradores mais atraentes do que no passado (Kerr et al., 2014). Durante a mais recente retomada do Capital de Risco Corporativo, por volta de 2010, os Aceleradores Corporativos apareceram como um novo fenômeno ao lado de seus pares não corporativos. Hochberg (2015) e Crichton (2014) apontam que isso foi desencadeado por empresas que estavam mais ansiosas do que no 
passado, para estarem próximas de empreendimentos que aumentam seu próprio potencial de inovação.

Heinemann (2015) classifica como corporativo um programa de aceleração em que $50 \%$ ou mais de sua propriedade estiver vinculada a uma corporação cujo negócio principal não seja trabalhar com Startups, e quando as diretrizes do programa forem derivadas dos objetivos da corporação que o mantém. As aceleradoras corporativas (AC) pertencem a uma ou mais corporações, têm seus objetivos derivados das corporações responsáveis e oferecem programas para Startups (Heinemann, 2015). Além disso, para o mesmo autor, no mesmo ano, os programas das aceleradoras corporativas possuem processo seletivo intermitente, buscam Startups em estágio inicial ou de crescimento, têm prazo limitado de duração e podem exigir participação societária. As aceleradoras corporativas são um tipo de programa de inovação aberta que visa internalizar tecnologias e, em contrapartida, oferecem apoio, recursos financeiros, espaço físico, entre outros (Weiblen; Chessbrough, 2015). Ribeiro, Plonski e Ortega (2015), por outro lado, caracterizaram as aceleradoras corporativas como oferta de investimento financeiro com contrapartida ligada à propriedade da empresa. Os Aceleradores Corporativos podem ser uma forma pela qual as empresas diversificarem seus gastos em negócios externos. Bradford (2014) descreve os Aceleradores Corporativos como apenas mais um meio a partir do qual as empresas financiam as empresas em estágio inicial, causadas pelo recente aumento do Capital de Risco Corporativo.

Um AC busca atrair e formar um grupo de Startups sucinto, promissor e adepto aos interesses de uma empresa patrocinadora que, por um período de tempo pre-determinado, propõe mecanismos de apoio que, normalmente, incluem rodadas de financiamento, auxílio com profissionais e empreendedores experientes, e espaços de trabalho compartilhado (Hochberg, 2015; Weiblen; Chesbrough, 2015). Pelo apoio que recebem, as Startups participantes, geralmente, oferecem quotas da empresa ou direitos de exploração conjunta da propriedade intelectual desenvolvida. Porém, esse fator não é uma regra: existem várias PACs que não requisitam direitos de participação ou propriedade, especialmente porque há componentes estratégicos no interesse das corporações (formação de fornecedores, por exemplo) (Bernthal, 2013; Dempwolf; Auer; D’ippolito, 2014; Lehmann, 2014; Weiblen; Chesbrough, 2015; Dee et al., 2015).

Hochberg (2015) mencionou os Aceleradores Corporativos, descrevendo-os como um certo tipo de acelerador que é "iniciado" por corporações e, muitas vezes, é semelhante aos aceleradores regulares. No entanto, o autor alertou que alguns Aceleradores Corporativos também exibem características atípicas e em evolução. Sua pesquisa também destaca que os programas são movidos pelo desejo da corporação de aumentar sua capacidade de inovação e melhorar sua visibilidade em tecnologias futuras (Hochberg, 2015). 


\section{MÉTODO}

Propõe-se, neste trabalho, o desenvolvimento de uma revisão sistemática da literatura sobre os assuntos "Corporate Venture Capital" e "Acelerador Corporativo", a fim de traçar suas semelhanças e diferenças. A revisão sistemática é desenvolvida com o objetivo de responder a um problema específico e utiliza-se de métodos explícitos e sistemáticos para identificar, selecionar e avaliar, criticamente, os estudos acerca da temática escolhida pelos autores, e para a coleta e análise de dados dos estudos incluídos na revisão (Rother, 2007). Dentre os diferentes métodos de revisão sistemática da literatura, optou-se neste trabalho pela revisão integrativa, pois tal modalidade permite o desenvolvimento de estudos de revisão com um escopo muito mais amplo do que as demais modalidades, integrando publicações de cunho teórico ou empírico, pautadas em abordagens quantitativas ou qualitativas.

A revisão integrativa permite resumir o passado da literatura, de modo que se alcance uma compreensão mais abrangente de um fenômeno em particular, apresentando-se o estado da técnica e permitindo a contribuição ao desenvolvimento teórico da área estudada com a geração de novos conhecimentos, assim como com implicações para as práticas organizacionais (Botelho; Cunha; Macedo, 2011; Mendes; Silveira; Galvão, 2008; Whittemore; Knafl, 2005).

Após o estabelecimento do tema de pesquisa, a primeira decisão tomada foi relativa à definição da problemática de pesquisa, sendo estabelecido a seguinte questão: "Quais as diferenças e similitudes dos institutos da Corporate Venture Capital e dos Aceleradores Corporativos?".

Em um segundo momento foram pesquisados os termos "Corporate Venture Capital" " "Aceleradores Corporativos" nas bases de dados EBSCO, Web of Science, e Scopus.

Na base de dados Web of Science, usando o termo ("Corporate Venture Capital”), entre parênteses e com aspas, teve-se o retorno de 115 (cento e quinze) documentos, no total. Limitando estes documentos apenas para artigos, teve-se o retorno de 90 (noventa) artigos, sendo apenas 6 (seis) abertos. Lendo o resumo destes artigos abertos, apenas 4 (quatro) deles mostraram relevante para a pesquisa. Após leitura completa destes artigos, apenas 1 (um) mostrou-se relevante.

Pesquisando o título dos artigos fechados, foi detectado pela leitura dos resumos destes artigos que apenas 38 (trinta e oito) dos 84 (oitenta e quatro) artigos eram relevantes para a pesquisa. Após leitura ampla dos artigos, identificou-se que 26 (vinte e seis) eram relevantes. No total, foram considerados 27 (vinte e sete) artigos relevantes para essa pesquisa.

Já o termo "Aceleradores Corporativos", pesquisado na língua inglesa: ("Corporate Accelerators") entre aspas e dentro de parênteses, apresentou, no total, 9 (nove) documentos, sendo 7 (sete) artigos, dos quais 1 (um) apresentou-se aberto e relevante para a pesquisa. Pesquisando o título dos artigos fechados, foi detectado pela leitura dos resumos destes artigos fechados, que apenas 2 (dois) dos 6 (seis) artigos eram relevantes para a pesquisa. Após leitura dos artigos, por inteiro, identificou-se 1 (um) relevante, totalizando a pesquisa em 1 (um) artigo relevante. 
Pesquisando os dois termos "Corporate Accelerators" e "Corporate Venture Capital" juntos (AND), com aspas e entre parênteses, retornaram 5 (cinco) artigos, no total de documentos, mostrando apenas 1 (um) artigo com relevância. As outras formas de pesquisa, com estes termos juntos (sem parênteses, termos dentro das mesmas aspas), não apresentaram resultados relevantes. Totalizando, nesta etapa de busca, então, tivemos apenas 1 (um) artigo relevante.

- $\quad$ No total, a base de dados Web of Science colaborou com 29 (vinte e nove) artigos relevantes para o desenvolvimento do presente trabalho.

Na base de dados Scopus, usando o termo ("Corporate Venture Capital”), entre parênteses e com aspas, teve-se o retorno de 1.369 (mil trezentos e sessenta e nove) documentos, no total. Limitando estes documentos apenas para artigos, teve-se o retorno de 78 (setenta e oito) artigos, sendo apenas 4 (quatro) abertos. Lendo o resumo destes artigos abertos, apenas 1 (um) deles mostrou-se relevante. Pesquisando o título dos artigos fechados, foi detectado pela leitura dos resumos destes artigos que apenas 24 (vinte e quatro) dos 74 (setenta e quatro) artigos, eram relevantes para a pesquisa. Após leitura dos artigos, por inteiro, identificou-se que 11 (onze) eram relevantes, totalizando, 12 (doze) artigos relevantes.

Com o termo ("Corporate Accelerators"), entre aspas e dentro de parênteses, apresentou-se o total de 34 (trinta e quatro) documentos, sendo 15 (quinze) artigos, dos quais 1 (um) apresentou-se aberto e relevante para o trabalho. Pesquisando o título dos artigos fechados, foi detectado pela leitura dos resumos destes artigos que 11 (onze) dos 14 (catorze) artigos eram relevantes para a pesquisa. Após leitura ampla dos artigos, identificou-se apenas 2 (dois) relevantes para o desenvolvimento do trabalho, totalizando 3 (três) artigos relevantes.

Pesquisando os dois termos "Corporate Accelerators" e "Corporate Venture Capital”, juntos (AND), com aspas e entre parênteses, apresentaram 5 (cinco) documentos no total, sendo 2 (dois) artigos fechados. Pesquisando o título destes artigos fechados, foi detectado pela leitura dos resumos que os 2 (dois) artigos eram relevantes para a pesquisa. Em um segundo momento, com leitura ampla destes artigos, apenas 1 (um) mostrou-se relevante. As outras formas de pesquisa, com estes termos juntos (sem parênteses, termos dentro das mesmas aspas), não apresentaram resultados. Totalizou-se 1 (um) artigo relevante.

- No total, a base de dados EBSCO colaborou com 16 (dezesseis) artigos relevantes para o desenvolvimento do presente trabalho.

$\mathrm{Na}$ base de dados EBSCO, usando o termo ("Corporate Venture Capital”), entre parênteses e com aspas, teve-se o retorno de 1.229 (mil duzentos e vinte e nove) documentos no total. Limitando estes documentos apenas para artigos, teve-se o retorno de 56 (cinquenta e seis) artigos, sendo apenas 27 (vinte e sete) abertos. Lendo o resumo destes artigos abertos, apenas 12 (dose) deles mostraram-se relevante. Pesquisando o título dos artigos fechados, foi detectado pela leitura dos resumos destes artigos que apenas 4 (quatro) dos 29 (vinte e nove) artigos eram relevantes para a pesquisa.

Com o termo ("Corporate Accelerators"), entre aspas e dentro de parênteses, apresentou-se o total de 51 (cinquenta e um) documentos, sendo 39 (trinta e nove 
artigos, dos quais 12 (dose) abertos e relevantes para o trabalho. Pesquisando o título dos artigos fechados, foi detectado pela leitura dos resumos destes artigos, que 11 (onze) dos 14 (catorze) artigos eram relevantes para a pesquisa. Após leitura ampla dos artigos, identificaram-se apenas 2 (dois) relevantes para o desenvolvimento do trabalho, totalizando 3 (três) artigos relevantes.

Pesquisando os dois termos "Corporate Accelerators" e "Corporate Venture Capital" juntos (AND), com aspas e entre parênteses, apresentaramu-se 65 (sessenta e cinco) documentos no total, sendo 22 (vinte e dois) artigos fechados. Pesquisando o título destes artigos fechados foi detectado, pela leitura dos resumos, que os 13 (treze) artigos eram relevantes para pesquisa. Em um segundo momento, com leitura ampla destes artigos, apenas 10 (dez) mostraram-se relevantes. As outras formas de pesquisa, com estes termos juntos (sem parênteses, termos dentro das mesmas aspas), não apresentaram resultados.

A partir da coleta dessas informações dos artigos estudados é que se desenvolveu a análise crítica da produção científica sobre Corporate Venture Capital e Corporate Accelerators, o que permitiu apontar suas semelhanças e diferenças, apresentadas na seção a seguir.

\section{APRESENTAÇÃO DOS RESULTADOS}

A partir da coleta de dados dos artigos, pôde-se evidenciar consenso e dissonâncias entre autores, tanto a respeito do tema Corporate Venture Capital como Aceleradores Corporativos, em diferentes aspectos. Os quadros 1 e 2 mostram, de forma sucinta, as principais discordância entre os autores, em cada um dos constructos pesquisados.

\section{DISCUSSÃO E RESULTADOS}

Após análise dos artigos quanto à Corporate Venture Capital (CorpVC), tem-se como consenso que as empresas que recebem investimentos são Startups e que elas adotam a lógica de investimento de CorpVC devido a objetivos não só financeiros como, também, estratégicos, sendo, assim, uma maneira de complementar as atividades de inovação da empresa (seja por meio de absorção de capacidades que estas Startups possuem ou por práticas de Open Innovation, por exemplo) ou um meio de adquirir e/ou "criar" novas capacidades (Chesbrought, 2000, 2002; CHESbrought; Tucci, 2004; Dushnitsky; Shapira, 2010; Hellman, 1996). O fato de o CorpVC ser baseado em objetivos estratégicos, e não só financeiros, o diferencia das outras modalidades de investimentos feitas diretamente em empresas, como por exemplo o Private Equity.

Quanto à análise dos artigos referentes ao índice de sucesso em níveis financeiros, a estrutura de CorpVC tem sido associada a histórias de fracasso (Fast, 1978; Rind, 1981; Sykes; Gompers, 2002; Baird; Rasmussen, 2002) devido à baixa performan- 


\begin{tabular}{|c|c|c|}
\hline \multicolumn{3}{|c|}{ Corp VC } \\
\hline Definição por: & $\begin{array}{l}\text { Chesbrought, 2000, 2002; } \\
\text { Chesbrought; Tucci, 2004; Dushnitsky; } \\
\text { Shapira, 2010; Hellman, } 1996\end{array}$ & $\begin{array}{l}\text { Fast, 1978; Rind, 1981; Sykes; } \\
\text { Gompers, 2002; Baird; Rasmussen, } \\
2002\end{array}$ \\
\hline O que é & Fundo de Investimento Privado & Fundo de Investimento Privado \\
\hline Objetivo & $\begin{array}{l}\text { Realizar investimentos em projetos de } \\
\text { inovação (Startups) }\end{array}$ & $\begin{array}{l}\text { Fomentar empreendimentos } \\
\text { que promovam negócios } \\
\text { complementares, ou que } \\
\text { alavanquem as vendam } \\
\text { de seus produtos }\end{array}$ \\
\hline $\begin{array}{l}\text { Atrativo para a } \\
\text { Startup }\end{array}$ & $\begin{array}{l}\text { Investimento financeiro para } \\
\text { alavancagem do modelo de negócio }\end{array}$ & $\begin{array}{l}\text { Investimento financeiro para } \\
\text { alavancagem do modelo de negócio }\end{array}$ \\
\hline $\begin{array}{l}\text { Espaço físico } \\
\text { interno }\end{array}$ & Não & Não \\
\hline Duração & Indeterminado & Indeterminado \\
\hline Seleção & Direta & Direta \\
\hline $\begin{array}{l}\text { Pagamento dos } \\
\text { custos }\end{array}$ & Sim & Sim \\
\hline Investimento & Obrigatório & Obrigatório \\
\hline $\begin{array}{l}\text { Internalização } \\
\text { da Inovação }\end{array}$ & Não & Mencionado, mas não necessário \\
\hline $\begin{array}{l}\text { Estágio da } \\
\text { Startup }\end{array}$ & Final & Final \\
\hline
\end{tabular}

Fonte: Os autores.

ce, quando comparado aos fundos de Venture Capital (VC). Parte da justificativa viria do maior foco nos objetivos estratégicos vis-à-vis com os objetivos financeiros. Entretanto, a literatura e estes fundos reportam muitas outras razões para essa percepção, como por exemplo: 1. Falta de experiência e sofisticação; 2 . Lentidão nas decisões de investimento; 3. Inconsistência no suporte às empresas investidas; 4. Potencial conflito de interesses entre os objetivos estratégicos da empresa-mãe e a empresa investida; 5. Políticas de compensação e rotatividade de empregados; 6 . Políticas internas; 7. Objetivos que geram conflitos; 8. Gestão incompetente (Fast, 1978; Rind, 1981; Sykes; Gompers, 2002; Baird; Rasmussen, 2002).

Por essas questões, alguns estudos afirmam que os CorpVCs ganharam muita experiência ao trabalhar lado a lado com os fundos de VC independente. As pesqui- 
Quadro 2: Mapa de autores AC

\begin{tabular}{|c|c|c|c|}
\hline & & $A C$ & \\
\hline Definição por: & Shankara, 2018 & Christensen,2015 & Dempwolf, 2014 \\
\hline O que é & Programa & Programa & Modelo de negócio \\
\hline Objetivo & $\begin{array}{l}\text { Catalisar Startups e } \\
\text { buscar inovação para } \\
\text { empresa madura }\end{array}$ & $\begin{array}{l}\text { Catalisar Startups e } \\
\text { buscar inovação para } \\
\text { empresa madura }\end{array}$ & $\begin{array}{l}\text { Catalisar Startups para } \\
\text { seguir para a fase de } \\
\text { investimentos }\end{array}$ \\
\hline $\begin{array}{l}\text { Atrativo para a } \\
\text { Startup }\end{array}$ & Mentoria, network & Mentoria, network & Mentoria, network \\
\hline $\begin{array}{l}\text { Espaço físico } \\
\text { interno }\end{array}$ & $\begin{array}{l}\text { Mencionado, mas não } \\
\text { necessário }\end{array}$ & Não mencionado & $\begin{array}{l}\text { Mencionado, mas não } \\
\text { necessário }\end{array}$ \\
\hline Duração & $\begin{array}{l}\text { Prazo fixo, mínimo } 3 \\
\text { (três) meses }\end{array}$ & $\begin{array}{l}\text { Prazo fixo, mínimo } 3 \\
\text { (três), máximo } 6 \text { (seis) } \\
\text { meses }\end{array}$ & $\begin{array}{l}\text { Prazo fixo, menor que } 12 \\
\text { (doze) meses }\end{array}$ \\
\hline Seleção & Coorte & Coorte & Coorte \\
\hline $\begin{array}{l}\text { Pagamento dos } \\
\text { custos }\end{array}$ & $\begin{array}{l}\text { Mencionado, mas não } \\
\text { necessário }\end{array}$ & Sim & Sim \\
\hline Investimento & $\begin{array}{l}\text { Mencionado, mas não } \\
\text { necessário }\end{array}$ & $\begin{array}{l}\text { Mencionado, mas não } \\
\text { necessário }\end{array}$ & Sim \\
\hline $\begin{array}{l}\text { Internalização } \\
\text { da Inovação }\end{array}$ & Sim & Sim & Sim \\
\hline $\begin{array}{l}\text { Estágio da } \\
\text { Startup }\end{array}$ & Inicial & Inicial & Inicial \\
\hline
\end{tabular}

Fonte: Os autores.

sas apontam que CorpVCs, agora, enfatizam uma abordagem mais similar àquela adotada pelos fundos, na qual eles investem com o objetivo financeiro, tentando maximizar o valor financeiro do investimento, especialmente depois da fase de seleção e investimento (Gompers, 2002). Com base nos artigos pesquisados, constatou-se que empresas estabelecidas em muitos setores, geralmente, mantêm CorpVC como resultado do investimento em várias Startups (Basu et al., 2011). Os relacionamentos com CorpVC representam uma importante fonte de conhecimento para as empresas consolidadas, pois esta estabelece laços de fronteiras com Startups, que buscam novas tecnologias ou estruturam novos modelos de negócios (Dushnitsky; Lenox, 2005). As empresas, frequentemente, realizam investimentos em CorpVC para captar novas tecnologias (Dushnitsky, 2006). Os laços de CorpVC influenciam 
o desempenho de inovação de uma empresa investidora, aumentando a quantidade e a variedade de conhecimento disponíveis, maximizando os seus esforços de inovação (Dushnitsky; Lenox, 2005; Wadhwa; Kotha, 2006). Esses fluxos são promovidos pela avaliação, monitoramento e colaboração do investidor com seu portfólio de empreendimentos (Dushnitsky; Lenox, 2005). Pesquisas também mostram que os investidores do CorpVC exibem variação na diversidade de seus investimentos, não focando necessariamente em Startups cujo objeto se assemelha com o conhecimento já abarcado da empresa investidora (Basu et al., 2011; Lin; Lee, 2011).

Muitos aspectos de um relacionamento CorpVC facilitam o acesso ao conhecimento de uma Startup. Em primeiro lugar, antes de investir, as empresas normalmente realizam due diligence em vários aspectos, gestores, plano de negócios, finanças, mercados-alvo, produtos e tecnologia (Dushnitsky, 2006). Muitas vezes, há uma unidade de negócios na empresa investidora envolvida na avaliação da tecnologia e dos produtos desenvolvidos pela Startup em potencial (Dushnitsky, 2006; Basu et al., 2011).

O estudo da literatura sobre AC permitiu identificar importantes constatações quanto às origens e à definição do construto, às fases de desenvolvimento das pesquisas acerca do tema, assim como das principais características dirigidas a esta proposta, conforme se verá a seguir.

Para agir de forma empreendedora dentro da organização, ou seja, perceber mudanças, identificar/construir novas oportunidades potenciais e explorar essas potencialidades, algumas corporações investem em atividades de ACs (Shankara, 2018). Por perceberem que é caro, incerto e lento confiar apenas nos esforços internos de inovação para competir com empresas empreendedoras ágeis, que perturbam o mercado (Christensen et al., 2015), ACs têm estabelecido como objetivo principal permitir que Startups aperfeiçoem o produto, focalizem mercados-alvo, encontrem maneiras de atrair novos usuários e clientes da empresa já consolidada devido à evolução de seu modelo de negócio. Assim, AC deve ser vista como a capacidade de uma corporação já consolidada catalisar o percurso da Startup, disponibilizando clientes, investidores, tecnologias, redes sociais e mentores em um período mais curto, de modo a mudar sua taxa de escala ou sua direção estratégica.

Três aspectos do processo de aceleração corporativa são cruciais para o sucesso das Startups e das empresas consolidadas: (1) acesso a clientes, (2) revisões periódicas, (3) orientação (Shankara, 2018). Richter (2017) aponta que os objetivos da utilização de ACs variam de empresa para empresa, no entanto, é consenso entre as pesquisas que o objetivo primordial é a necessidade de alavancar a formação de novas parcerias e redes de investimentos para o desenvolvimento real dos produtos e serviços já oferecidos ou, até mesmo, para a descoberta de novos mercados. A maioria das empresas em cooperação com Startups via AC parece ser especialmente interessada em melhorar sua capacidade geral de inovação, embora a imagem da empresa consolidada também seja um subproduto útil. Os resultados do caso geral dos aceleradores sugerem que eles podem ser um programa útil para empresas que 
buscam melhorar seu desempenho inovador. Os processos de seleção competitiva, funções predeterminadas e expectativas atribuídas a Startups, um período de tempo definido, rede e educação obrigatórias, e a estruturação desses programas em lotes ou coortes introduzem alta pressão e reconhecimento precoce de falhas (Richter, 2017).

No geral, como mostra o Quadro 3, CorpVC e os ACs têm elementos em comum, como sua propriedade, público-alvo e, até certo ponto, seus objetivos. As diferenças são principalmente o montante do financiamento fornecido, o forte foco dos ACs a apoio não monetário, e a estrutura de tempo fixa baseada em coorte. ACs geralmente visam Startups em fase inicial, enquanto CorpVC visa Startups em fase final, como demonstra o Gráfico 1.

Quadro 3: Comparativo Corp VC e AC

\begin{tabular}{|c|c|c|}
\hline Indicador & Corporate Venture Capital & Aceleradora Corporativa \\
\hline Proprietário & Empresas estabelecidas & Empresas estabelecidas \\
\hline Objetivos & $\begin{array}{l}\text { Financeiros e/ou estratégico; } \\
\text { exploração e aquisição de } \\
\text { conhecimento. }\end{array}$ & $\begin{array}{l}\text { Estratégico. Exploração, ganhar } \\
\text { vantagens competitivas. }\end{array}$ \\
\hline Origem dos Objetivos & Derivados da Empresa & Derivados da Empresa \\
\hline $\begin{array}{l}\text { Portfólio de } \\
\text { Empresas }\end{array}$ & $\begin{array}{l}\text { Startups em estágio intermediários } \\
\text { ou avançados }\end{array}$ & Startups em estágios iniciais \\
\hline Apoio às Startups & Primeiramente monetário & $\begin{array}{l}\text { Primeiramente, rede de contatos } \\
\text { e experiência }\end{array}$ \\
\hline $\begin{array}{l}\text { Duração } \\
\text { prédeterminada }\end{array}$ & Não & Sim (máximo 12 meses) \\
\hline Entrada & Em caráter contínuo & Baseado em turmas \\
\hline Seleção & Competitiva & Competitiva \\
\hline Financiamento & Sim & Pode ocorrer \\
\hline Participação acionária & Sim & Pode ocorrer \\
\hline $\begin{array}{l}\text { Participação na } \\
\text { sociedade }\end{array}$ & Minoritária & Minoritária \\
\hline Surgimento & Anos 1960 & Anos 2010 \\
\hline
\end{tabular}

Fonte: Heinemann, 2010 adaptado pelos autores. 
Gráfico1: Estágios do AC e Corp VC

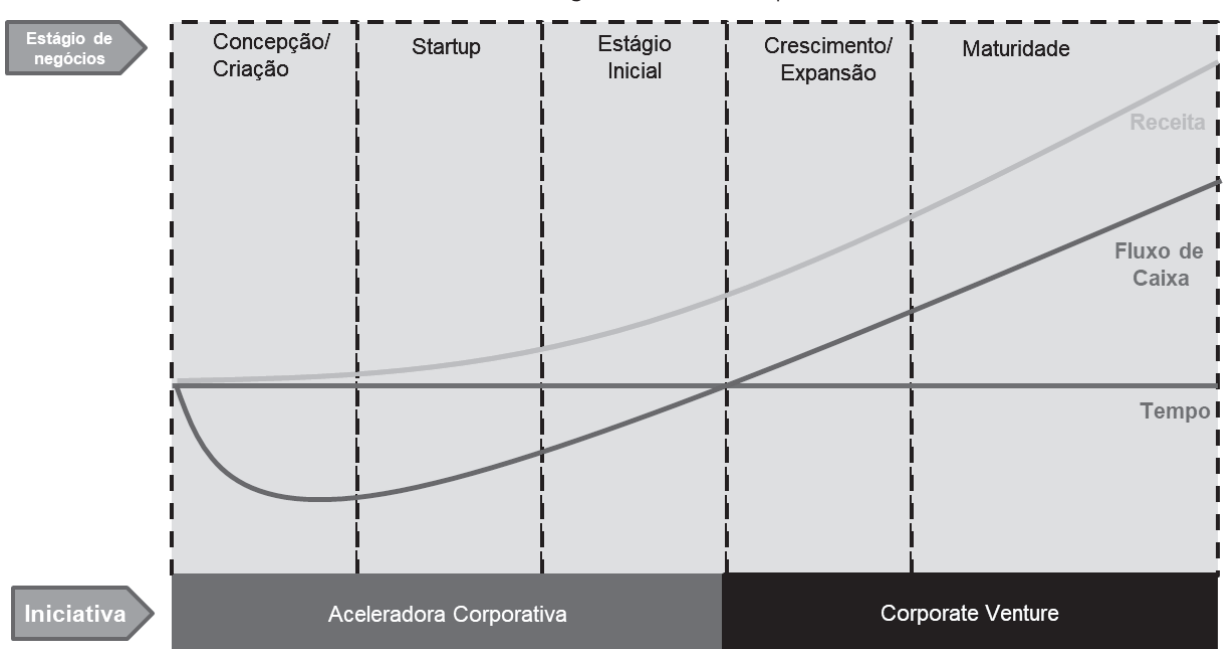

Fonte: Elaborado pelos autores.

Em ambos, AC e CorpVC, os empreendimentos acelerados estão fora da corporação, podendo, em algumas situações, existir programas de ACs com foco em internalizar as Startups financiadas. Os objetivos das duas metodologias mostram similitudes, ambas visam melhorar a capacidade da empresa de inovar, experimentar novas tecnologias e atingir a ambidestria de nível empresarial (BASU et al., 2011). No entanto, ACs são diferentes de CorpVC quanto à internalização da inovação investida, nesta, a finalidade de se aventurar em empresas externas consiste em criar novos empreendimentos fora do limite organizacional (Covin; Miles, 2007) enquanto, na outra, o objetivo é internalizar a inovação. CorpVC envolvem, predominantemente, o investimento de capital de uma empresa (Miles; Covin, 2002; Zahra et al., 2016); já as ACs, raramente assumem um risco patrimonial. Quanto à estrutura jurídica, enquanto a CorpVC baseia-se em contratos e, geralmente, requer um objetivo comum entre os parceiros, Startup e empresa investidora (Schildt et al., 2005), as ACs não exigem o estabelecimento de metas ou contratos comuns entre a corporação e o empreendimento. Finalmente, enquanto a intenção de CorpVC é criar (e alcançar) retorno financeiro e estratégico comum (entre a corporação e o empreendimento) (Schildt et al., 2005), nas ACs a corporação pode buscar resultados diferentes daqueles esperados na estruturação do programa.

\section{CONCLUSÃO}

A pesquisa apontou que o fenômeno de Aceleradores Corporativos e de Corporate Venture Capital é uma prática de busca por inovação, que pode ser semi-institucionalizada em empresas que têm a intenção de firmar parcerias em ambientes externos, sobretudo em Startups. 
A principal conclusão desta pesquisa é uma lista de "nortes" para a melhor utilização dos mecanismos de captação de inovação estudados por empresas maduras.

Após a análise, notou-se que é consenso que as empresas que recebem investimentos, via CorpVC e AC, são Startups e que elas adotam a lógica de investimento devido a objetivos não só financeiros como, também, estratégicos, sendo, assim, uma maneira de complementar as atividades de inovação da empresa (seja por meio de absorção de capacidades que esta Startup possui ou por práticas de Open Innovation, por exemplo) ou um meio de adquirir e/ou "criar" novas capacidades.

$\mathrm{O}$ fato de CorpVC e AC serem baseados em objetivos estratégicos, e não só financeiros, os diferenciam das outras modalidades de investimentos feitas diretamente em empresas, no entanto, percebeu-se que ambas as estratégias têm diferenças cruciais, que, se vistas anteriormente pela empresa madura, podem ser mais bem aplicadas, garantindo o índice de sucesso maior, baseado no propósito e objetivo da Corporação financiadora.

\section{REFERÊNCIAS BIBLIOGRÁFICAS}

Bernthal,, Brad. Investment Accelerators. Entrepreneurial Bus. 2013 Black, B. S., \& Gilson, R. J. Venture capital and the structure of capital markets: banks versus stock markets. Journal of financial economics, v. 47, n. 3, p. 243-277, 1998.

Baird, Ross, Lily Bowles, and Saurabh Lall (2013). Bridging the "Pioneer Gap": The Role of Accelerators in Launching High-Impact Enterprises. Aspen Network of Development Entrepreneurs and Village Capital.

Barclays (2014). Barclays Plc Annual Report 2014. url: http: //www.home.barclays/cont ent/ dam/barclayspublic/docs/InvestorRelations/AnnualReports/AR2014/Barclays_PLCAnnualReport_\%20 2014. pdf.

Bliemel, Martin J. et al. (2013). Accelerate Australia Far: Exploring the Emergence of Seed Accelerators within the Innovation Ecosystem Down-Under. SSRN Scholarly Paper ID 2422173. Rochester, NY: Social Science Research Network. url: http: //papers.ssrn. com/abstract= 2422173.

Bradford, Jon (2014). Corporate-run startup accelerators: the good, the bad and the plain ugly Tech.eu. url: http: //tech. eu/f eatures/779/corporate-run-startup-acceleratorsgood- bad-plain-ugly/

CHESBROUGH, H. Making Sense of Corporate Venture Capital. Harvard Business Review, v.80, n. 3, p. $90-7,2002$.

Chesbrough, H., \& Tucci, C. L. (2002). Corporate Venture Capital in the Context of Corporate Innovation.

Chesbrough, Henry W (2002). "Making Sense of Corporate Venture Capital”. In: Harvard Business Review 80.3, pp. 90-99. issn: 00178012. url: http: //libproxy.mit. edu/login?url= http: //search. ebscohost. com/login. aspx? direct=true $\& \mathrm{db}=\mathrm{bth} \& \mathrm{AN}=6327068 \&$ site=ehost-live

Christiansen, Jed (2011). Looking back - 1.5 years since "Copying Y Combinator". per aspera ad astra. url: http: //blog. jedchristiansen. com/2011/02/23/looking-back-1-5-years-since-copying-y-combinator/

Clark, Patrick (2013). Waiting for the Accelerator Bubble to Pop. BloombergView. url: http: //www. bloomberg. com/bw/articles/2013-03- 14/waiting-f or-the-acceleratorbubble-to-pop

Crichton, Danny (2014). Corporate Accelerators Are An Oxymoron. TechCrunch. Disponível em: <http:/social.techcrunch. com/2014/08/25/corporate-accelerators-are-an-oxymoron/> Acesso em: 09/ Nov/2018.

Dee, Nicola; Gill, David; Weinberg, Caren; McTavish, Stewart. What's the Difference. Nesta. 2015. 
Dempwolf, C. Scott, Jennifer Auer, Michelle D’Ippolito. (2014) Innovation Accelerators: Defining Characteristics Among Startup Assistance Organizations. Small Business Administration (SBA.

Dushnitsky, Gary and J. Myles Shaver (2009). "Limitations to interorganizational knowledge acquisition: the paradox of corporate venture capital".

Dushnitsky, Gary and Michael J. Lenox (2005). "When do firms undertake R\&D by investing in new ventures?” In: Strategic Management Journal 26.10, pp. 947-965. issn: 0143-2095, 1097-0266. doi: 10. 1002/smj. 488. (Visited on 03/21/2015). - (2006). "When does corporate venture capital investment create firm value?".

Ernst \& Young (2013). EY G20 Entrepreneurship Barometer 2013. Ernst \& Young. url: https: //webforms. ey. com/Publication/vwLUAssetsPI/TheEYG20_Entrepreneurship_Barometer_2013/\$FILE/ EY-G20-main-report. pdf (visited on 06/22/2015).

Gunday, G., Ulusoy, G., Kilic, K., \& Alpkan, L. (2011). Effects of innovation types on firm performance. International Journal of Production Economics, 133(2), 662-676. http://dx.doi.org/10.1016/j. ijpe.2011.05.014.

Heinemann, F. (2015). Corporate Accelerators: A Study on Prevalence, Sponsorship, and Strategy (Doctoral dissertation). Massachusetts Institute Of Technology. Cambridge, Massachusetts, Estados Unidos, 33-36.

Hochberg, Yael V (2015). “Accelerating Entrepreneurs and Ecosystems: The Seed Accelerator Model”. In: Innovation Policy and the Economy. Washington, DC. url: http: //www. nber. org/confer/2015/ IPEs15/Hochberg.pdf.

Hoffman, D. L., \& Radojevich-Kelley, N. (2012). Analysis of Accelerator Companies: An Exploratory Case Study of Their Programs, Processes, and Early Results. Small Business Institute Journal, 8(2), 54-70.

Kerr, William R., Ramana Nanda, and Matthew Rhodes-Kropf (2014). Entrepreneurship as Experimentation. Working Paper 20358. National Bureau of Economic Research. url: http: //www.nber. org/papers/w20358 (visited on 06/22/2015).

Kohler, T. (2016). Corporate accelerators: Building bridges between corporations and Startups. Business Horizons, 59(3), 347-357.

Lehmann, Peter. Corporate Accelerators: Characteristics and Motives. Dissertação [Mestrado]. Copenhagen Business School, 97 p. 2014.

Mocker, V.B., Bielli, S., \& Haley, C. (2015). Winning together. A guide to successful corporate-startup collaborations. Nesta

Norris, A. (2014). Startups and older firms: which is more responsive to local economic changes? Monthly Labor Review, 1-2.

Ribeiro, A.T.V.B., Plonski, G.A., \& Ortega, L.M. (2015). Um fim, dois meios: aceleradoras e incubadoras no Brasil. XVI Congresso Latino-Iberoamericano de Gestão da Tecnologia. Porto Alegre, RS, Brasil.

Simoudis, Evangelos (2014). Using Corporate Incubators and Accelerators to Drive Disruptive Innovation. 13.ago.2014. Disponível em. Acesso em 26/out/2018.

Weiblen, T., \& Chesbrough, H.W. (2015). Engaging with Startups to enhance corporate innovation. California Management Review, 57(2), 66-90. 